\title{
PH-responsive PVDF Composite Membranes Blended with Amphiphilic Poly (methacrylic acid)-block-polydimethylsiloxane-poly (methacrylic acid)
}

\author{
Dapeng Liu' ${ }^{1, a}$, Haiye Wang ${ }^{1, b}$ and Chunju $\mathrm{He}^{2, \mathrm{c}}$ \\ ${ }^{1}$ College of Materials Science and Engineering, Donghua University, Shanghai 201620, China \\ ${ }^{2}$ The State Key Laboratory for Modification of Chemical Fibers and Polymer Materials, Donghua \\ University, Shanghai 201620, China \\ adp1573@163.com, b615434312@qq.com, chchunjuhe@dhu.edu.cn
}

Keywords: amphiphilic copolymer, PVDF membrane, pH-responsive, smart membrane.

\begin{abstract}
In this work, a well-defined amphiphilic triblock copolymer poly (methacrylic acid)-block-polydimethylsiloxane-poly (methacrylic acid) (PMAA-b-PDMS-b-PMAA) synthesized via atom transfer radical polymerization was employed for the preparation of $\mathrm{pH}$-responsive poly (vinylidene fluoride) (PVDF) membrane by facile blending method. The resultant blend membrane showed significantly $\mathrm{pH}$-responsive characteristics as confirmed by filtration experiments.
\end{abstract}

\section{Introduction}

Membrane technology has become one of the most important separation technologies over the past decades due to its simplicity in operation, low energy consumption, no chemical additives and so on. Nowadays, membrane technology has found their applications in almost every sector of industry including environmental, chemical, electric and biotechnological areas [1].

Stimuli-responsive membranes, that could self-regulate their permeability and characteristics in respond to small external changes such as $\mathrm{pH}$, temperature, ionic strength, electric field and photo-irradiation, have been drawing considerable attention continuously. Such smart membranes have the potential to be used in sensors and actuators, controlled release of chemicals or drugs, tissue engineering, water treatment and so on [2]. Compared with other external stimuli, $\mathrm{pH}$ is a crucial parameter which gives more choices both for the materials and for the application environment. Therefore, the development of $\mathrm{pH}$-responsive membranes is very meaningful both for science and technology [3].

In this work, novel amphiphilic copolymer PMAA-b-PDMS-b-PMAA was synthesized via atom transfer radical polymerization which is known to be an effective and convenient way for the synthesis of copolymer. The well-defined copolymer was employer for the preparation of $\mathrm{pH}$-responsive smart membranes via facile blending method. The chemical structures of the copolymers were detected by FTIR and ${ }^{1} \mathrm{H}$ NMR. The permeation properties and morphologies of the pure and blend membranes were also investigated in detail.

\section{Materials and Methods}

\subsection{Chemicals and Materials.}

Pentamethyldiethylenetriamine (PMDETA), polyvinylpyrrolidone (PVP, K30), tert-butyl methacrylate (tBMA), triethylamine (TEA), copper(I) bromide (CuBr, 98\%), tetrahydrofuran (THF), butanone, n-hexane, methanol, dichloromethane, N,N-dimethylacetamide (DMAc), calcium hydride $\left(\mathrm{CaH}_{2}\right)$, magnesium sulfate, trifluoroacetic acid (TFA, 99\%) and 2-bromoisobutyryl bromide (BIBB) were obtained from Sinopharm Chemical Reagent (Shanghai, China). Hydroxypropyl polydimethylsiloxane (HO-PDMS-OH, $\mathrm{Mn}=4000 \mathrm{~g} / \mathrm{mol}$, PDI $=1.41$ ) was purchased from Gelest Inc. THF and TEA were dried over $\mathrm{CaH}_{2}$ and distilled. $\mathrm{CuBr}$ was washed with acetic acid, ethanol and diethyl ether successively before use. All other chemicals were used as received. 


\subsection{Synthesis of Amphiphilic Block Copolymer PMAA-b-PDMS-b-PMAA.}

Firstly, macroinitiator Br-PDMS-Br was synthesized through the reaction of hydroxypropyl polydimethylsiloxane with 2-bromoisobutyryl bromide according to a method published previously [4].

Then triblock copolymer PtBMA-b-PDMS-b-PtBMA was synthesized via ATRP. A mixture of tBMA (3.98 mL, $25.00 \mathrm{mmol}$ ), Br-PDMS-Br macroinitiator (1.00 g, $0.25 \mathrm{mmol}$ ), butanone (13.2 mL) and PMDETA (100 $\mu \mathrm{L}, 0.50 \mathrm{mmol})$ were added to a Schlenk flask equipped with a magnetic stirring bar before subjected to three freeze-pump-thaw cycles. $\mathrm{CuBr}(0.072 \mathrm{~g}, 0.50 \mathrm{mmol})$ was then added under nitrogen atmosphere. The reaction was carried out at $60^{\circ} \mathrm{C}$ for $24 \mathrm{~h}$. After that, the solution was concentrated and precipitated in water. The final product was filtrated and lyophilized.

For a typical hydrolysis, $1.0 \mathrm{~g}$ PtBMA-b-PDMS-b-PtBMA and $1.5 \mathrm{~g}$ TFA were dissolved in 10.0 $\mathrm{mL}$ of dichloromethane. The solution was stirred at $25^{\circ} \mathrm{C}$ for $24 \mathrm{~h}$. After that, the solution was concentrated and percipitated into n-hexane. The final product was dried at $60{ }^{\circ} \mathrm{C}$ for $24 \mathrm{~h}$. The polymers were characterized by FTIR and ${ }^{1} \mathrm{H}$ NMR.

\subsection{Membrane Preparation.}

The PVDF membrane was prepared by nonsolvent induced phase separation method from a solution of $10.0 \mathrm{~g}$ PVDF, $4.0 \mathrm{~g}$ PEG, $50.0 \mathrm{~g}$ DMAc and a predetermined amount ( $0 \mathrm{~g}$ for the control membrane and $1.0 \mathrm{~g}$ for the blend membrane) of the amphiphilic copolymer. The solution was stirred at $60{ }^{\circ} \mathrm{C}$ for $12 \mathrm{~h}$ to form a homogenous solution before sealed and degassed over night at $60{ }^{\circ} \mathrm{C}$. Then the blend solution was casted on a glass plate with a casting knife of $200 \mu \mathrm{m}$ and immersed into a coagulation water bath at $25{ }^{\circ} \mathrm{C}$ immediately. After formation, the membrane was rinsed with deionized water extensively and stored in water before use.

\subsection{Membrane Characterization.}

Morphologies of the surface and cross-section of pure and blend membranes were observed by scanning electron microscopy (SEM, HITACHI S-3000, Japan). All of the membranes were freeze dried and sputter-coated with gold before test.

The $\mathrm{pH}$-responsive characteristics of the pure and blend membranes was characterized by the trans-membrane fluxes of buffer solutions. Buffer solutions with $\mathrm{pH}$ values of 3-8 prepared from 0.1 $\mathrm{M}$ citric acid and $0.2 \mathrm{M} \mathrm{Na}_{2} \mathrm{HPO}_{4}$ were used.A dead-end filtration system with an effective membrane area of $4.56 \mathrm{~cm}^{2}$ was employed to characterize the permeation property of the pure and blend membranes. Each membrane was first pressured at a constant trans-membrane pressure of 0.15 $\mathrm{MPa}$ for 30 min with buffer solutions. After the pressure was reduced to $0.10 \mathrm{MPa}$, the flux (J) was recorded and calculated by the following equation.

$$
\mathrm{J}=\mathrm{V} /(\mathrm{S} * \mathrm{t})
$$

where the parameters of $\mathrm{V}, \mathrm{S}$ and $\mathrm{t}$ denoted the buffer solution permeate volume (L), membrane area (m2) and permeation time (h) respectively.

\section{Results and Discussion}

\subsection{Copolymer Characterization.}

The structure of the triblock copolymer PtMBA-b-PDMS-b-PtMBA was confirmed by ${ }^{1} \mathrm{H}$ NMR spectra, as shown in Fig. 1 . The peak at $0.08 \mathrm{ppm}$ in the spectra can be assigned to the methyl protons in the PDMS segment and the peak at $1.43 \mathrm{ppm}$ is attributed to $-\mathrm{O}\left(\mathrm{CH}_{3}\right)_{3}$ in the PtMBA segment. Thus, PtMBA-b-PDMS-b-PtMBA is successfully synthesized.

The FTIR spectra of the copolymers are presented in Fig. 2. Compared with A, the broad absorbance from 2800 to $3600 \mathrm{~cm}^{-1}$ in B can be ascribed to carboxylic acid group [5] and the carbonyl stretch has shifted slightly. The peaks at $1399 \mathrm{~cm}^{-1}$ and $1375 \mathrm{~cm}^{-1}$ in A can be ascribed to tertiary butyl groups in PtMBA-b-PDMS-b-PtMBA. These two peaks are replaced by one peak at $1393 \mathrm{~cm}^{-1}$ which is attributed to methyl groups in the PDMS segment. All these results indicate that the amphiphilic copolymer PMAA-b-PDMS-b-PMAA is successfully synthesized. 


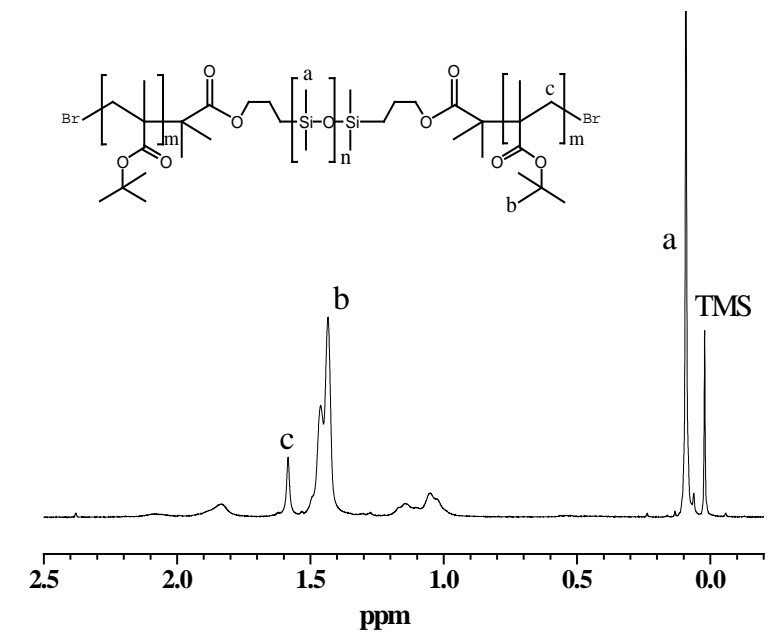

Fig. 1 1H NMR spectra for PtMBA-b-PDMS-b-PtMBA.

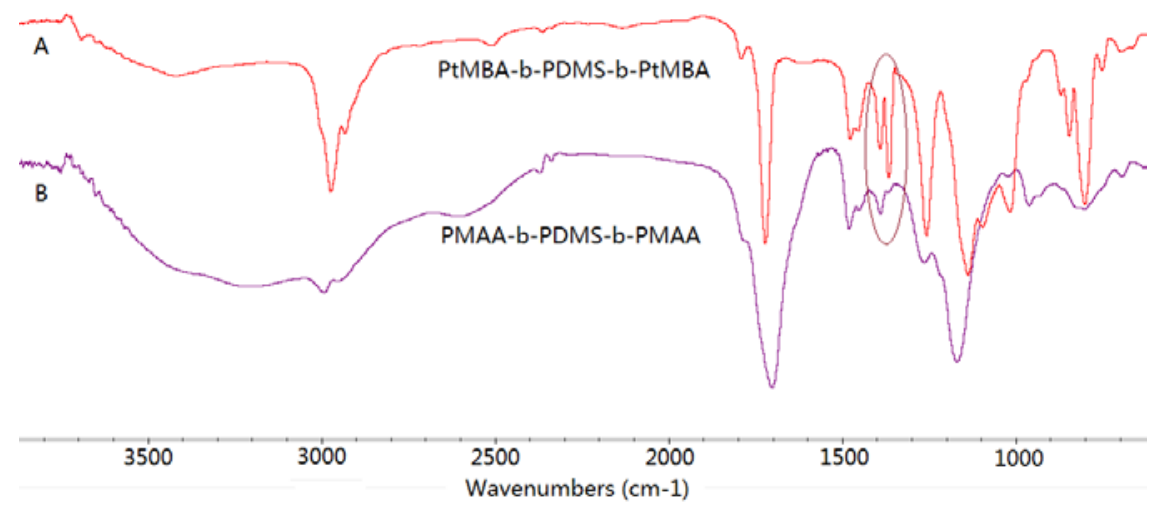

Fig. 2 FTIR of PtMBA-b-PDMS-b-PtMBA before (A) and after hydrolysis to PMAA-b-PDMS-b-PMAA (B).

\subsection{Membrane Characterization.}
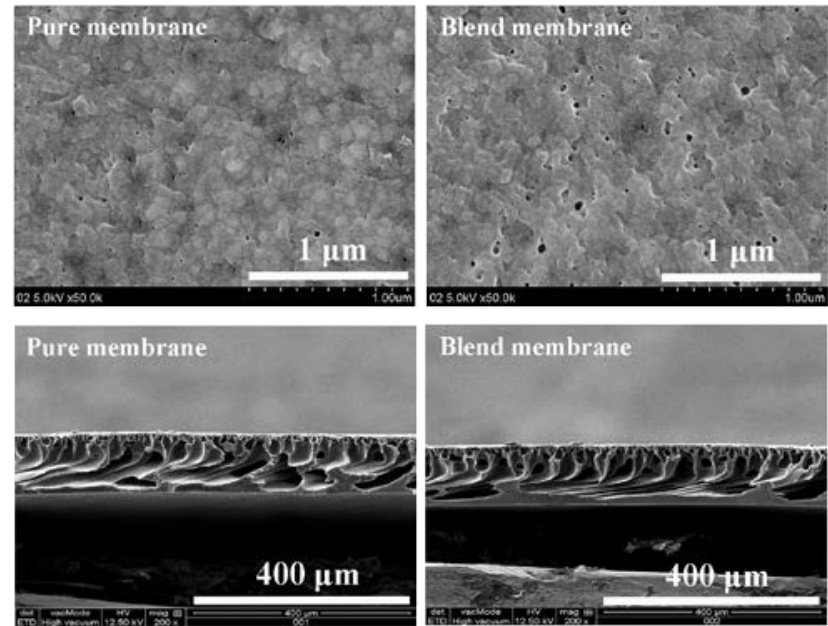

Fig. 3 SEM images of the pure and blend membranes.

SEM images of the surfaces and cross-sections of pure and blend membranes are presented in Fig. 3. Both of the membranes exhibit typical asymmetrical structures consisting of a dense top layer and a porous sub-layer. It can be seen from the surface images that addition of the amphiphilic copolymer into the casting solution results in an increase in pore size. However, there is no obvious difference between the cross-sectional morphologies of the pure and blend membrane.

The $\mathrm{pH}$-responsive properties of the membranes were characterized by filtration experiments. Fig. 4 shows the trans-membrane fluxes of buffer solutions with different $\mathrm{pH}$ values across the pure and blend membranes. It can be seen that fluxes of the pure PVDF membrane nearly do not change when 
the $\mathrm{pH}$ values the feed solution vary from 3 to 8 , indicating that the pure membrane does not have $\mathrm{pH}$-responsive characteristics. However, fluxes of the composite membrane blended with amphiphilic PMAA-b-PDMS-b-PMAA vary significantly from $575 \mathrm{~L} \cdot \mathrm{m}^{-2} \cdot \mathrm{h}^{-1}$ to $145 \mathrm{~L} \cdot \mathrm{m}^{-2} \cdot \mathrm{h}^{-1}$, which is negative correlated with the $\mathrm{pH}$ values of feed solution. This phenomenon can be explained by the conformational change of the PMAA segments of the amphiphilic copolymer on the pore channels. As we know, the hydrophilic PMAA segments will be enriched on membrane surface and pore channels through spontaneously surface segregation during the process of membrane formation. At high $\mathrm{pH}$ value, the carboxyl groups of the PMAA segments are dissociated and negatively charged. The repulsion between negative charged PMAA segments makes the segments extended, resulting in decreased pore size and decreased fluxes of the blend membrane.

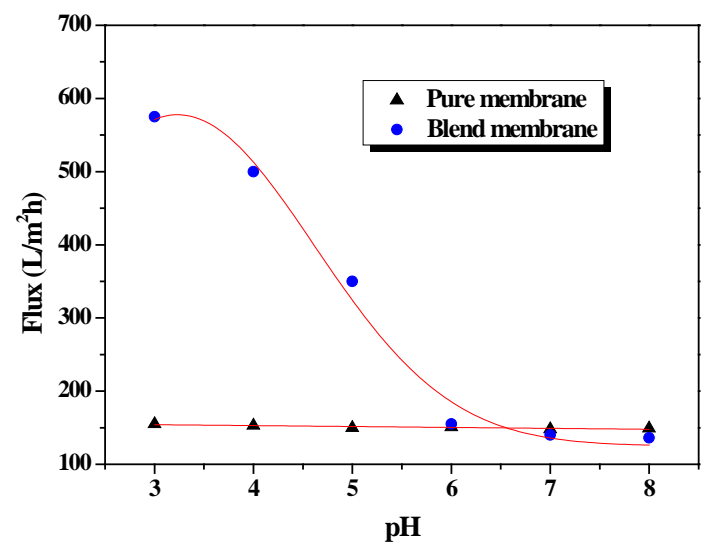

Fig. 4 Trans-membrane fluxes of buffer solutions with different $\mathrm{pH}$ values.

\section{Conclusion}

The well-defined PMAA-b-PDMS-b-PMAA was successfully synthesized via atom transfer radical polymerization and subsequent hydrolysis, which was employed for the modification of PVDF membrane via blending method. The blend membrane showed significantly pH-responsive characteristics due to conformational change of the PMAA segments of the amphiphilic copolymer on the pore channels.

\section{References}

[1]. Fu Liu, N. Awanis Hashim, Yutie Liu, et al. Progress in the production and modification of PVDF membranes. Journal of Membrane Science. Vol. 375 (2011) No. 1-2, p. 1-27.

[2]. Tao Luo, Shuo Lin, Rui Xie, et al. pH-responsive poly (ether sulfone) composite membranes blended with amphiphilic polystyrene-block-poly (acrylic acid) copolymers. Journal of Membrane Science. Vol. 450 (2014) No. 1, p. 162-173.

[3]. Changsheng Zhao, Shengqiang Nie, Min Tang, et al. Polymeric pH-sensitive membranes-a review. Progress in Polymer Science. Vol. 36 (2011) No. 11, p. 1499-1520.

[4]. Jianfeng Xu, Ming Qiu, Bomou Ma, et al. "Near perfect” amphiphilic conetwork based on end-group cross-linking of polydimethylsiloxane triblock copolymer via atom transfer radical polymerization. ACS Applied Materials \& Interfaces. Vol. 6 (2014) No. 17, p. 15283-15290.

[5]. Kelly A. Davis, Krzysztof Matyjaszewski. Atom transfer radical polymerization of tert-butyl acrylate and preparation of block copolymers. Macromolecules. Vol. 33 (2000) No. 11, p. 4039-4047. 